\title{
Enhanced wound healing via collagen-turnover-driven transfer of PDGF-BB gene in a murine wound model
}

\author{
Raj Kumar Thapa ${ }^{1}$, David J. Margolis², Kristi L. Kiick ${ }^{3 *}$, and Millicent O. Sullivan ${ }^{1 *}$ \\ ${ }^{1}$ Department of Chemical and Biomolecular Engineering, University of Delaware, Newark, DE \\ 19716 \\ ${ }^{2}$ Perelman School of Medicine, Department of Dermatology, University of Pennsylvania, \\ Philadelphia, PA 19104 \\ ${ }^{3}$ Department of Materials Science and Engineering, University of Delaware, Newark, DE 19716
}

*Corresponding authors:

Kristi L. Kiick, Department of Materials Science and Engineering, University of Delaware, Newark, DE 19716. Email: kiick@udel.edu

Millicent O. Sullivan, Department of Chemical and Biomolecular Engineering, University of

Delaware, Newark, DE 19716. Email: msullivan@udel.edu 
(A)

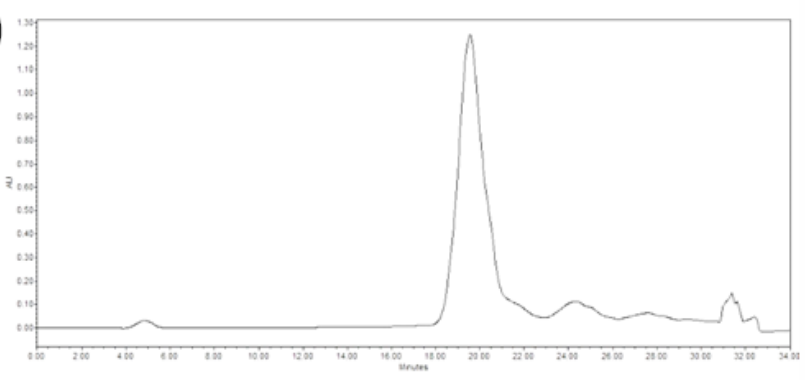

(B)

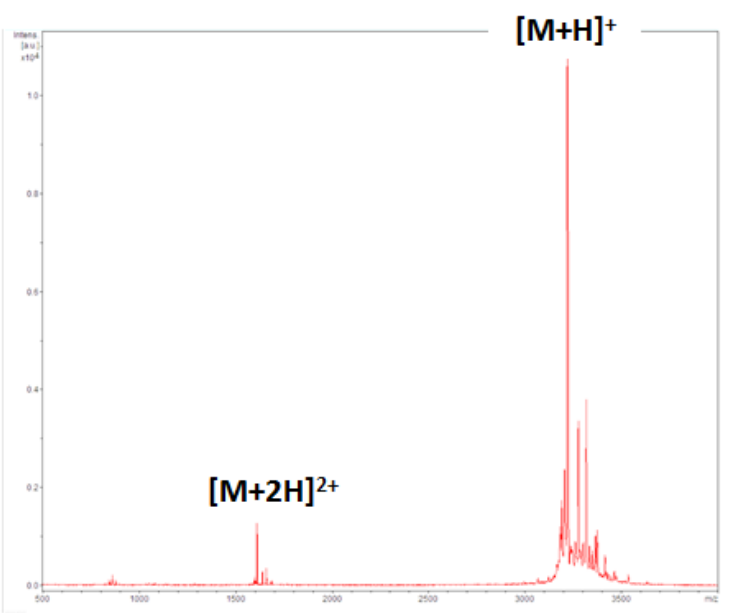

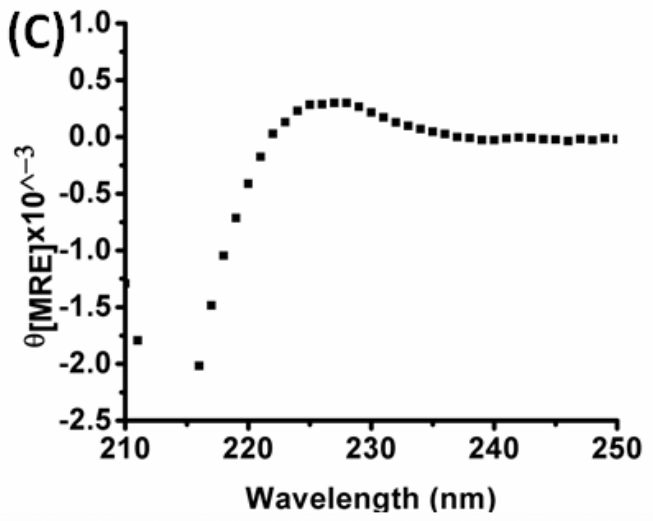

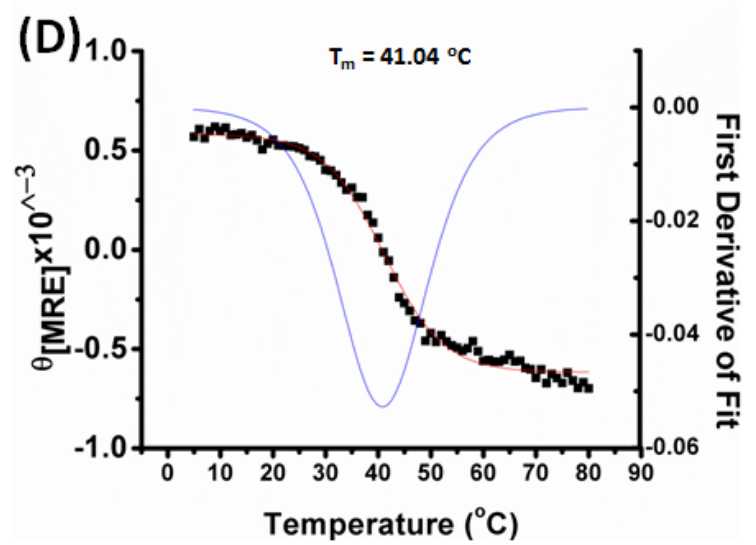

Figure S1: Characterization of synthesized CMPs. (A) HPLC chromatogram, (B) MALDI spectrum, and CD spectra for (C) wavelength and (D) temperature scans for the CMPs. 

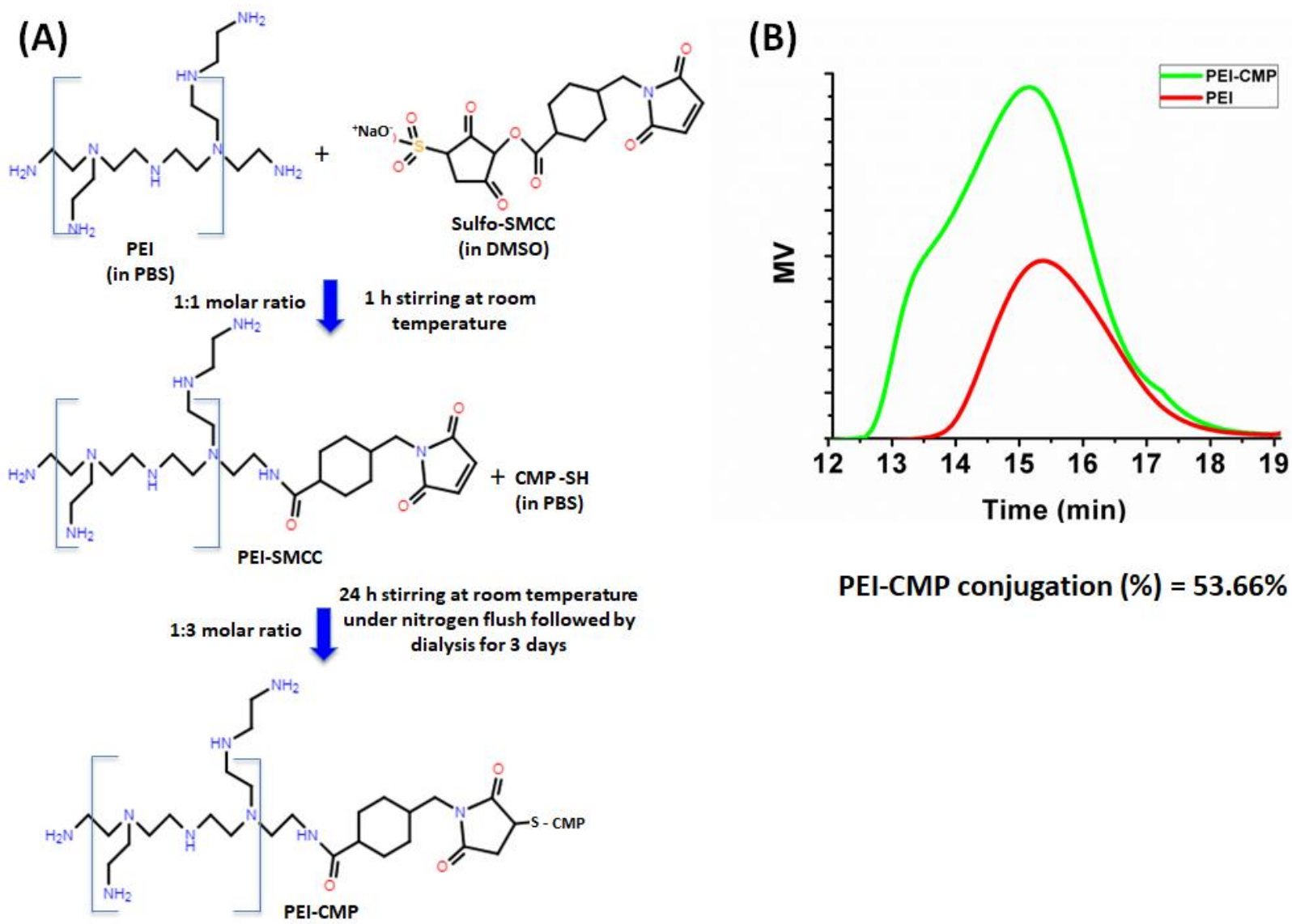

PEI-CMP conjugation $(\%)=53.66 \%$

Figure S2: Characterization of CMP-PEI conjugate. (A) Schematic representation of the reaction mechanism for CMP-PEI synthesis (a 1:1 molar ratio of PEI:Sulfo-SMCC and a 1:3 molar ratio of PEI-SMCC:CMP-SH were used for the reactions). (B) Gel permeation chromatography results for PEI and CMP-PEI conjugates. 
Non-lyophilized GLuc polyplexes

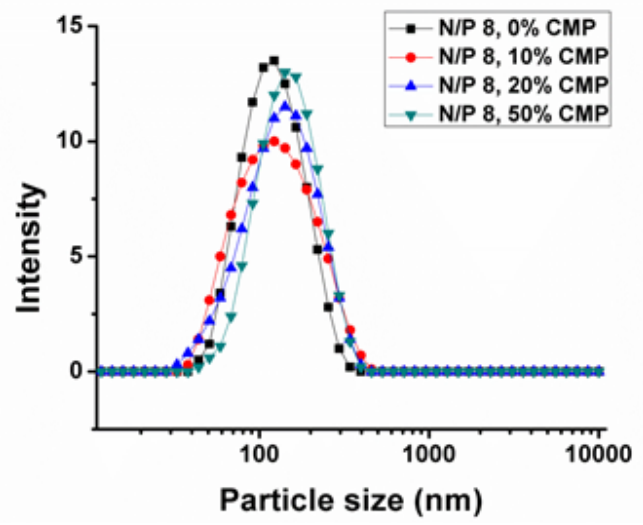

Lyophilized GLuc polyplexes

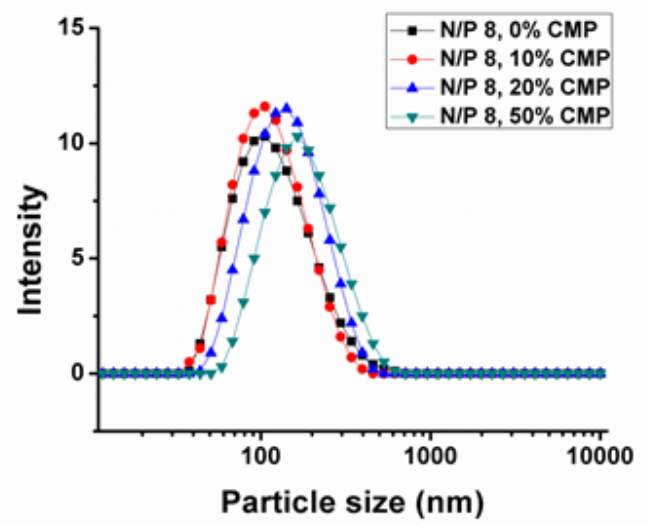

\begin{tabular}{|l|c|c|c|c|c|c|}
\hline \multirow{2}{*}{$\begin{array}{l}\text { GLuc polyplex } \\
\text { composition }\end{array}$} & \multicolumn{2}{|c|}{ Particle size (nm) } & \multicolumn{2}{c|}{ PDI } & \multicolumn{2}{c|}{ Zeta potential (mV) } \\
\cline { 2 - 7 } & Non-lyo & Lyo & Non-lyo & Lyo & Non-lyo & Lyo \\
\hline $0 \%$ CMP & $118.3 \pm 3.5$ & $117.6 \pm 2.7$ & $0.22 \pm 0.01$ & $0.23 \pm 0.01$ & $39.2 \pm 0.9$ & $42.8 \pm 3.6$ \\
\hline $10 \%$ CMP & $132.1 \pm 5.6$ & $136.2 \pm 2.0$ & $0.24 \pm 0.01$ & $0.23 \pm 0.02$ & $37.8 \pm 0.8$ & $38.6 \pm 1.3$ \\
\hline $20 \%$ CMP & $149.5 \pm 3.1$ & $175.8 \pm 5.9$ & $0.26 \pm 0.02$ & $0.27 \pm 0.01$ & $35.6 \pm 1.7$ & $34.9 \pm 2.2$ \\
\hline $50 \%$ CMP & $168.5 \pm 5.1$ & $190.4 \pm 6.3$ & $0.28 \pm 0.01$ & $0.29 \pm 0.01$ & $31.9 \pm 0.7$ & $31.8 \pm 1.3$ \\
\hline
\end{tabular}

Figure S3: Particle size measurements for GLuc polyplexes prepared in $20 \mathrm{mM}$ HEPES buffer (pH 6.0) measured using a Zetasizer. Intensity represents volume average for DLS measurements. Values in the table represent mean \pm standard deviation for a total of three separately prepared and analyzed samples. 

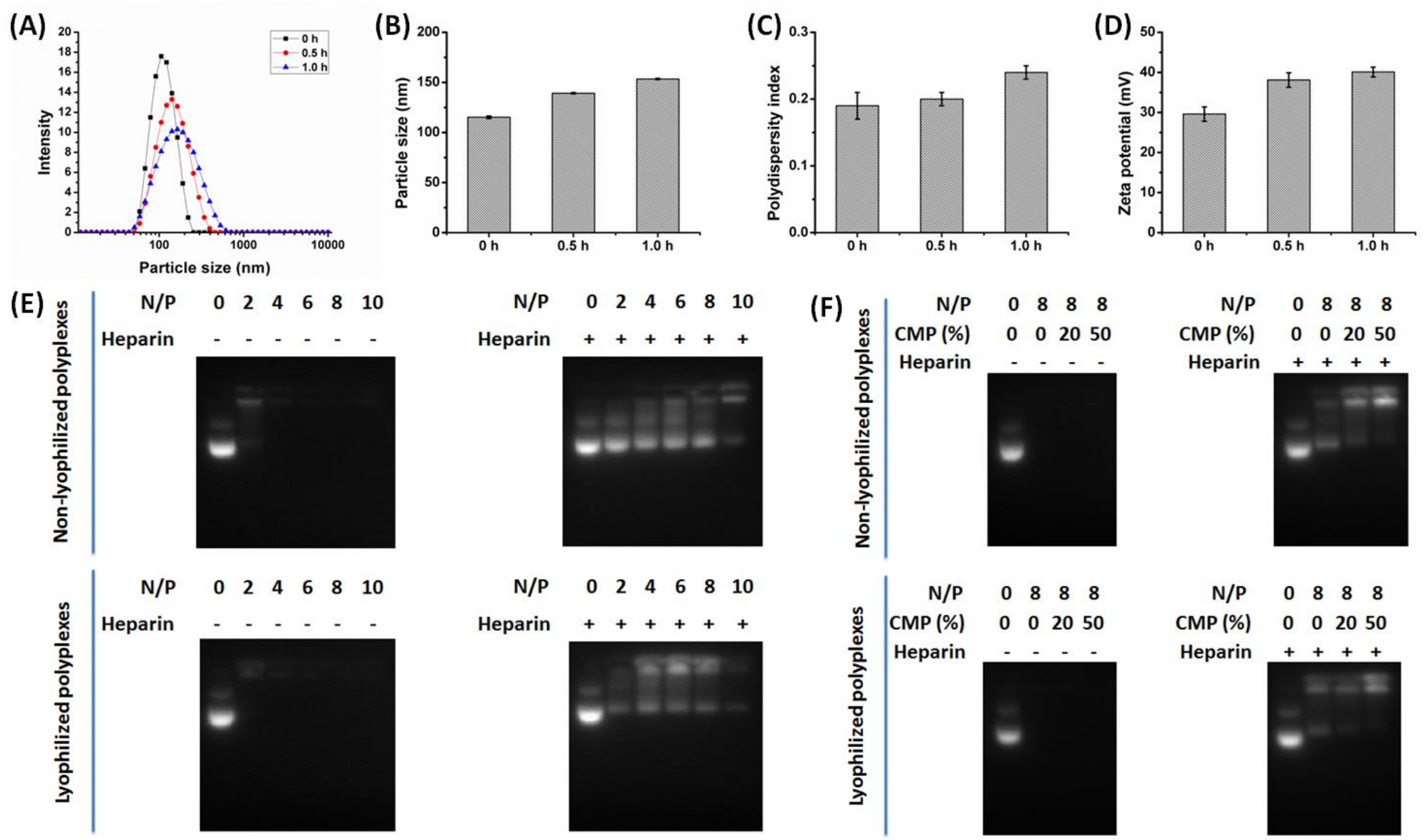

Figure S4: Determination of polyplex stability. (A, B) Particle size (intensity represents volume average for DLS measurements), (C) PDI, and (D) zeta potential measurements of PDGF polyplexes following incubation at $50{ }^{\circ} \mathrm{C}$ for up to $1 \mathrm{~h}$. Agarose gel electrophoresis of non-lyophilized and lyophilized polyplexes at different N/P ratios (E) and different CMP modifications (F). 


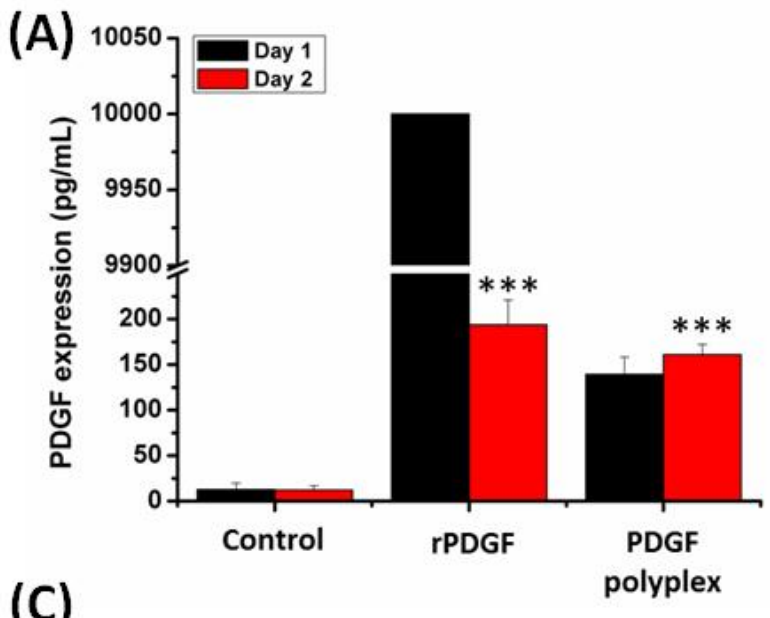

(C)
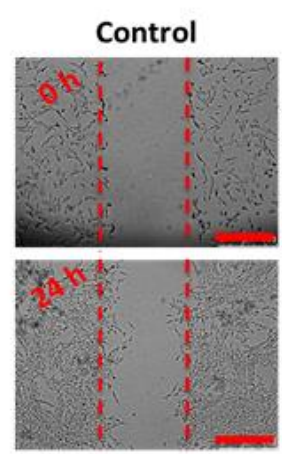

rPDGF

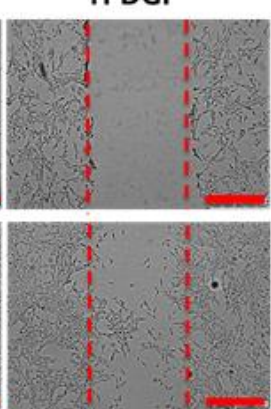

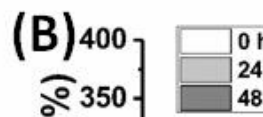

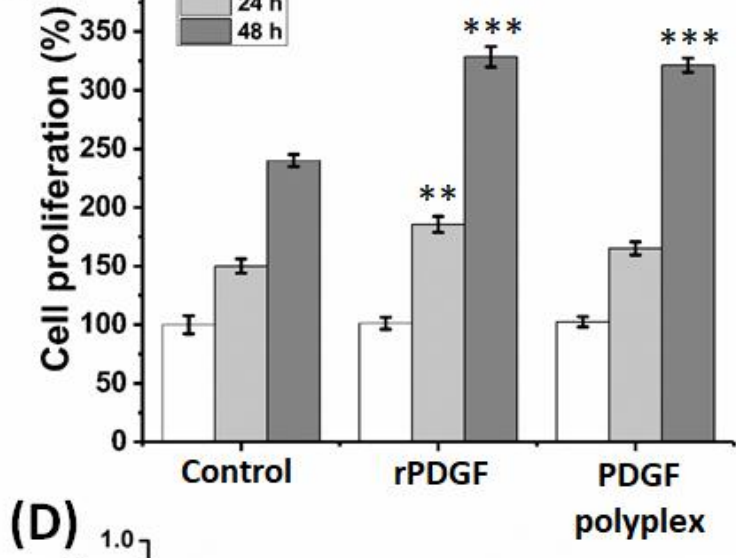

Figure S5: Effect of PDGF on NIH/3T3 cells. (A) PDGF expression following bolus transfection of NIH/3T3 cells with PDGF polyplexes. Effect of expressed PDGF on (B) cell proliferation and (C, D) migration. The data points represent mean \pm standard deviation for a total of three separately prepared and analyzed samples $(* \mathrm{P}<0.05, * * \mathrm{P}<0.01, * * * \mathrm{P}<0.001$ compared to control). 
(A)

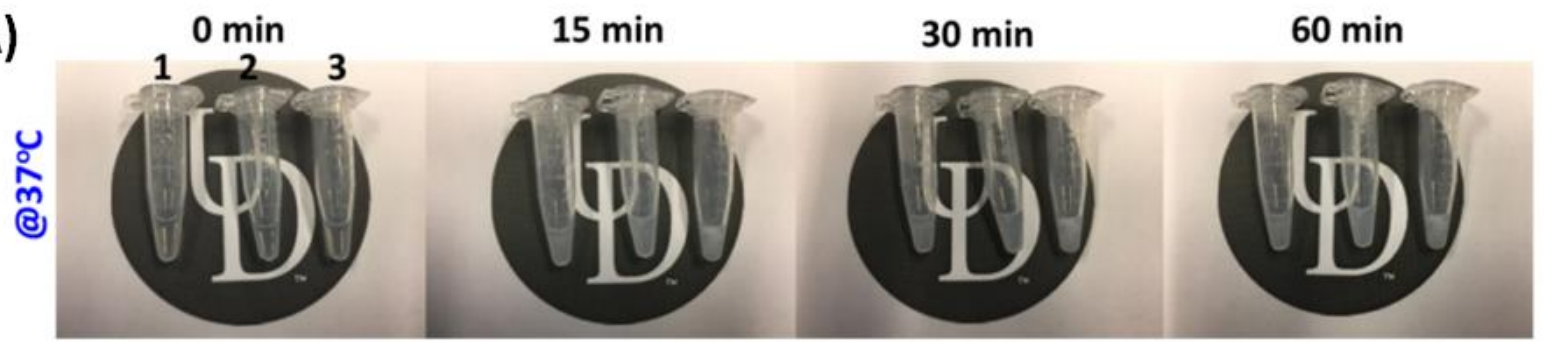

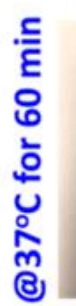

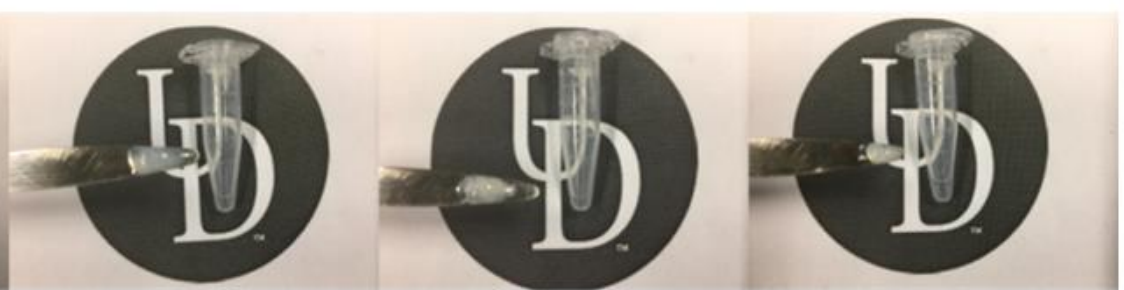

Co-gel compositions: 1: Collagen $2 \mathrm{mg} / \mathrm{ml}$, 2: Collagen $3 \mathrm{mg} / \mathrm{ml}$, 3: Collagen $4 \mathrm{mg} / \mathrm{ml}$ $+1.25 \mathrm{mg} / \mathrm{ml}$ Fibrinogen and $0.156 \mathrm{IU} / \mathrm{ml}$ Thrombin

(B)
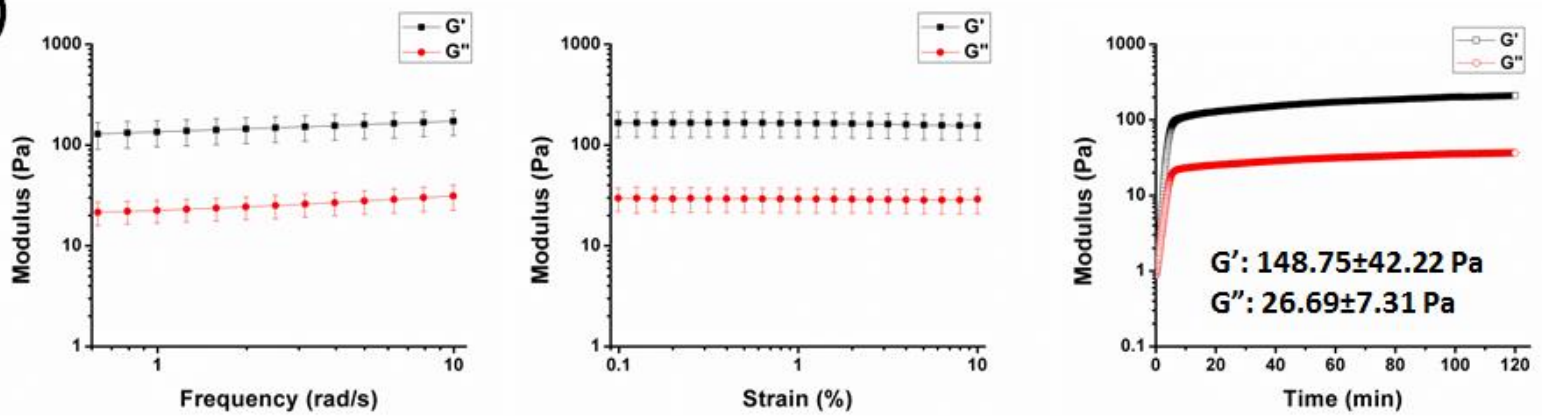

Figure S6: Characterization of co-gels. (A) Photographs of co-gels prepared with different concentrations of collagen. (B) Rheological measurements for co-gel prepared with a collagen concentration of $4 \mathrm{mg} / \mathrm{ml}$ (along with fibrinogen $1.25 \mathrm{mg} / \mathrm{mL}$ and thrombin $0.156 \mathrm{IU} / \mathrm{mL}$ ) at 37 ${ }^{\circ} \mathrm{C}$ determined using an DHR-3 rheometer. 

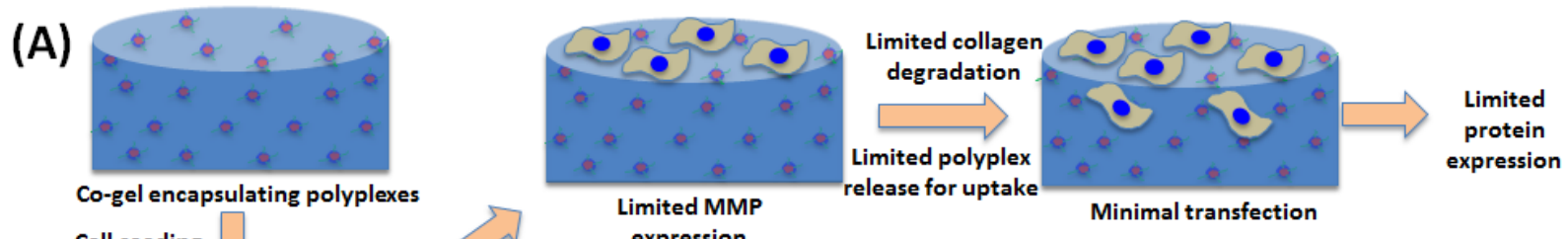
Cell seeding
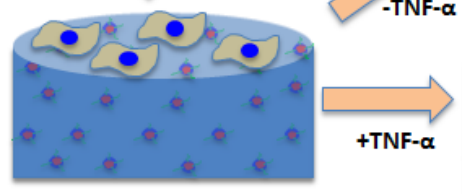

(B)
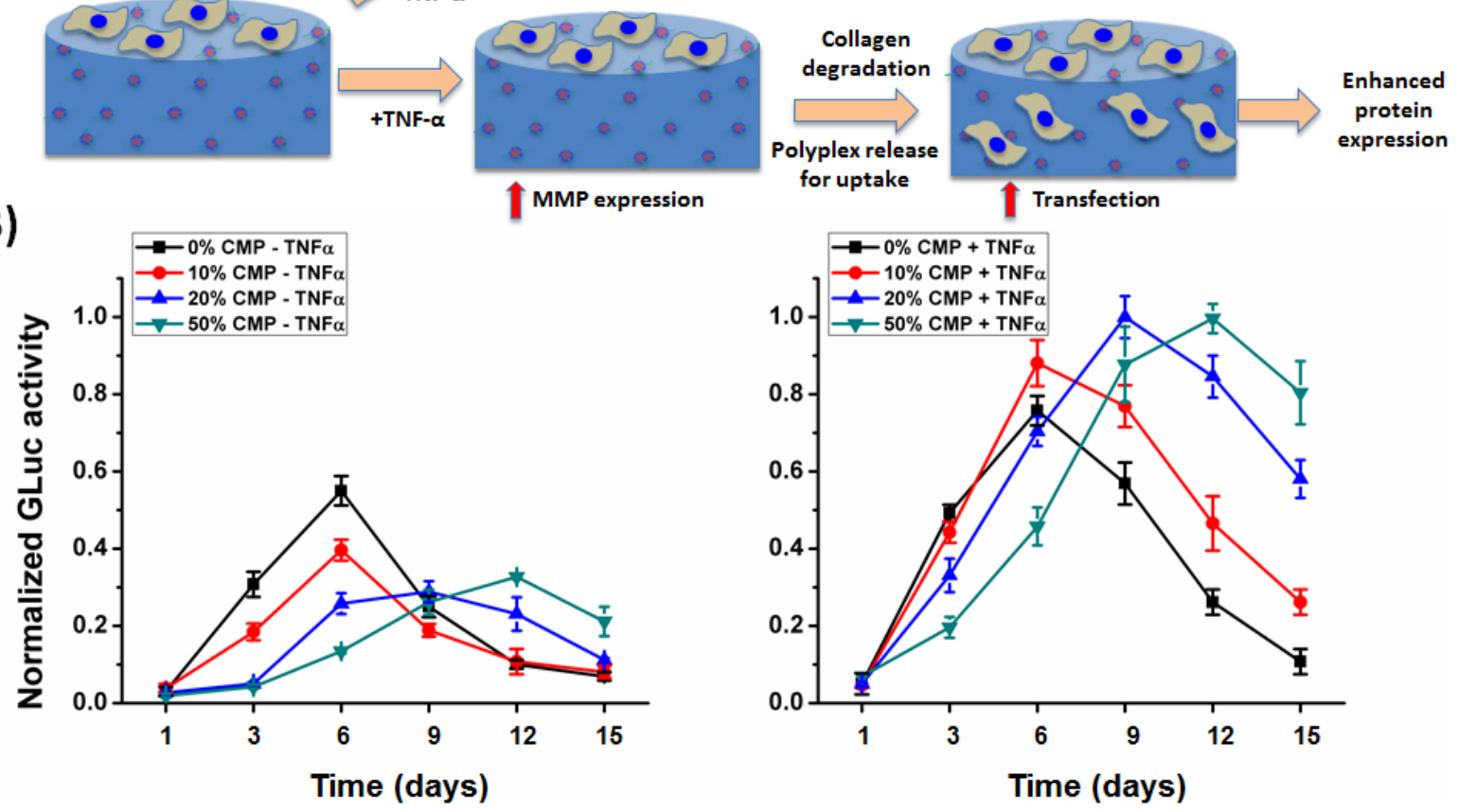

Figure S7: (A) Schematic representation of the procedure for co-gel-based GLuc transfection in the NIH/3T3 cells in the presence or absence of TNF- $\alpha$. (B) Normalized GLuc activity following fibroblast seeding over the GLuc polyplex-loaded co-gels at different time points. The data points represent mean \pm standard deviation for a total of three separately prepared and analyzed samples. In the absence of TNF- $\alpha$ treatment, statistical differences $(\mathrm{P}<0.05)$ in luciferase expression were observed for varying CMP modifications at different time points [i.e. (day 3: $0 \%$ and 10\%, 20\%, 50\% CMP co-gel; $10 \%$ and 20\%, 50\% CMP co-gel), (day 6: $0 \%$ and 10\%, 20\%, 50\% CMP cogel; $10 \%$ and 20\%, 50\% CMP co-gel; $20 \%$ and 50\% CMP co-gel), (day 9: $10 \%$ and 20\% CMP cogel); (day 12: $0 \%$ and 50\% CMP co-gel; $10 \%$ and 50\% CMP co-gel), (day 15: 0\% and 20\%, 50\% CMP co-gel; $10 \%$ and 50\% CMP co-gel)]. Furthermore, in the presence of TNF- $\alpha$ treatment, 
statistical differences $(\mathrm{P}<0.05)$ in luciferase expressions were observed for varying CMP modifications at different time points [i.e. (day 3: $0 \%$ and 20\%, 50\% CMP co-gel; $10 \%$ and 50\% CMP co-gel), (day 6: $0 \%$ and 50\% CMP co-gel; 10\% and 50\% CMP co-gel, 20\% and 50\% CMP co-gel), (day 9: $0 \%$ and 20\% CMP co-gel; $10 \%$ and 20\% CMP co-gel), (day 12: $0 \%$ and 20\%, 50\% CMP co-gel; $10 \%$ and 20\%, 50\% CMP co-gel), (day 15: 0\% and 10\%, 20\%, 50\% CMP co-gel; $10 \%$ and $20 \%, 50 \%$ CMP co-gel). 

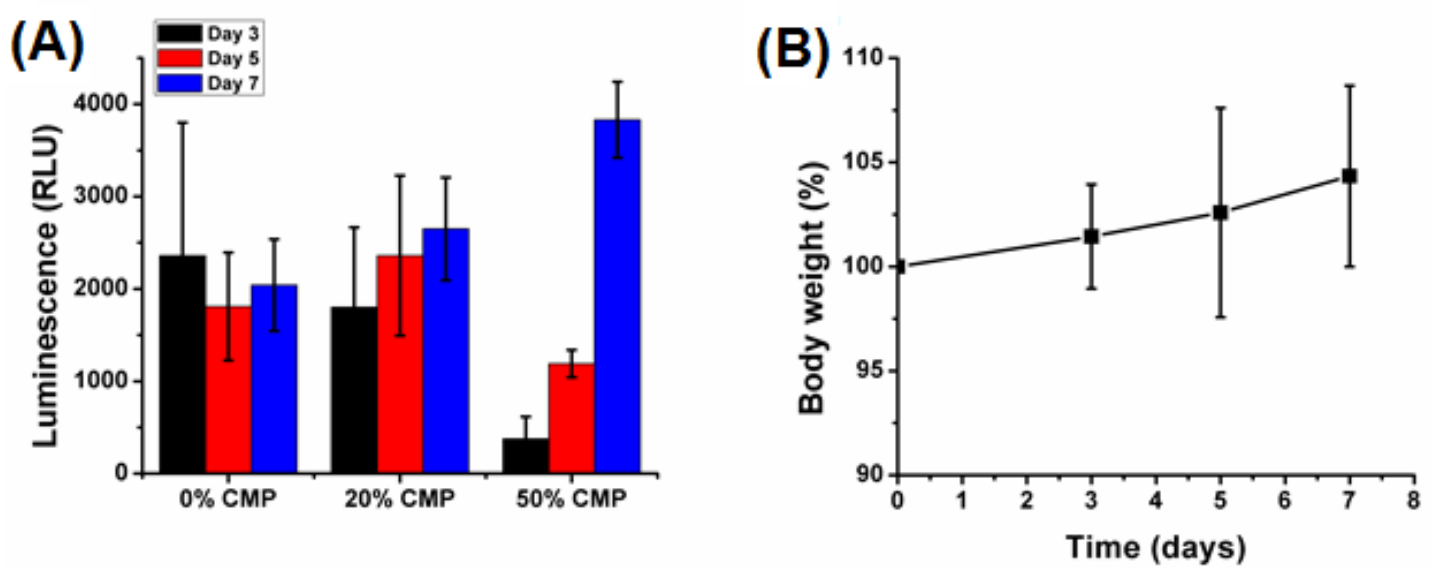

Figure S8: Evaluation of in vivo transfection efficiency using membrane bound luciferase polyplexes with different CMP modifications encapsulated in Matrigel. (A) Normalized luminescence for different CMP modified polyplex treatments for different time points. (B) Changes in body weight of mice. The data points represent mean \pm standard deviation for a total of three separately analyzed samples. 

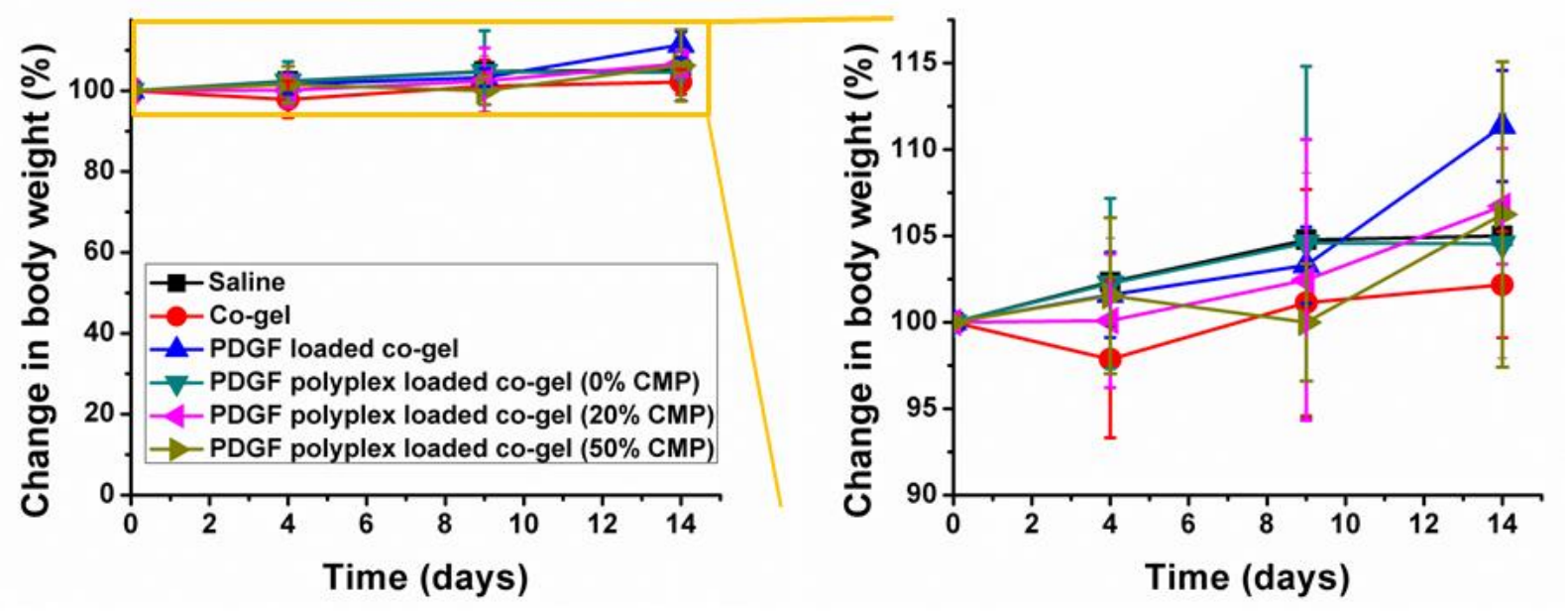

Figure S9: Body weights of mice treated with different formulations for up to 14 days. Each data point represents the mean \pm standard deviation for a total of three separate measurements. 


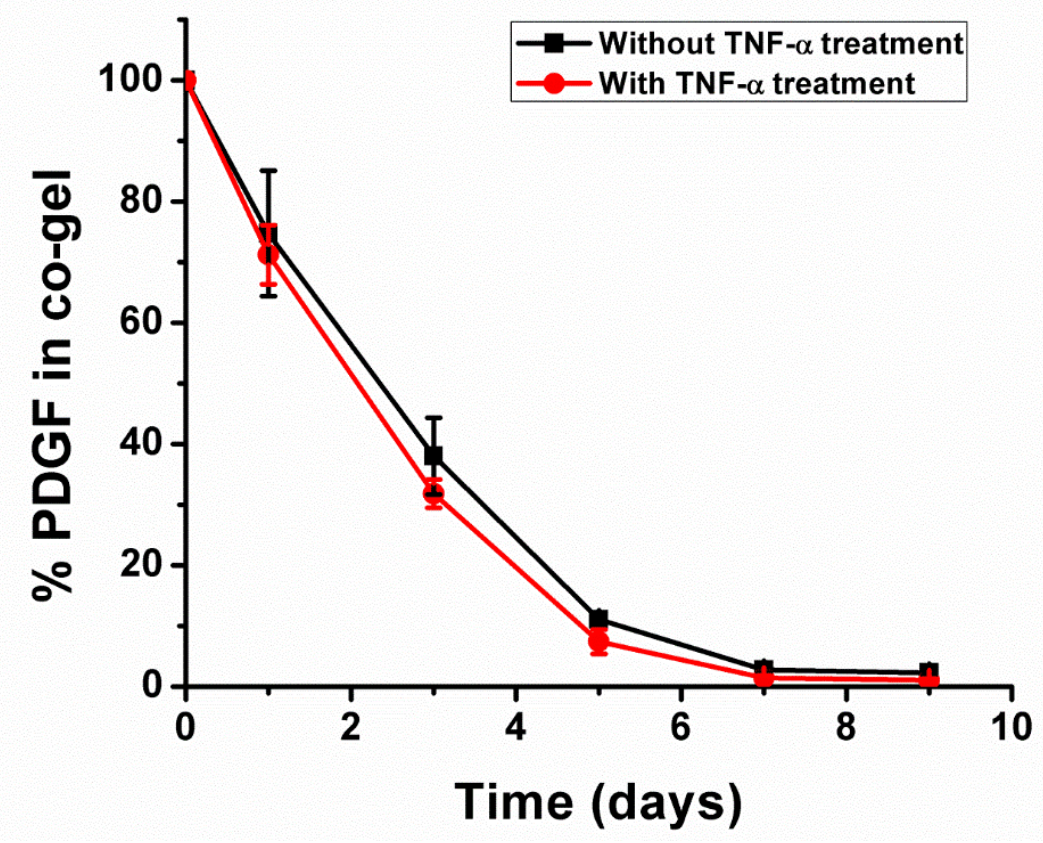

Figure S10: Stability of PDGF in co-gels seeded with NIH/3T3 cells. Percentage PDGF remaining in the co-gels with or without $\mathrm{TNF}-\alpha$ treatments. The data points represent mean \pm standard deviation for a total of three separately prepared and analyzed samples. 
Saline

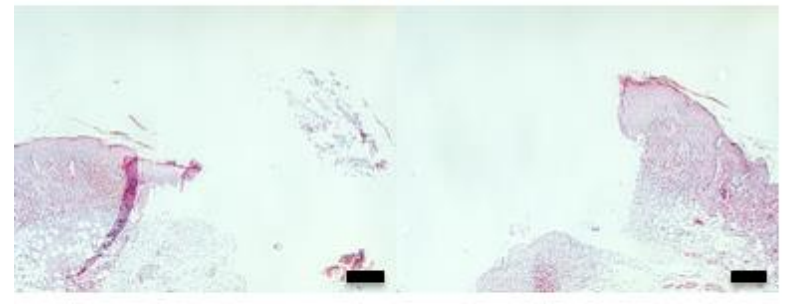

Blank co-gel

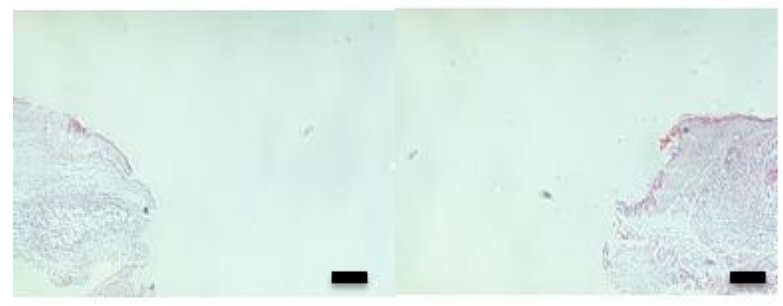

PDGF in co-gel

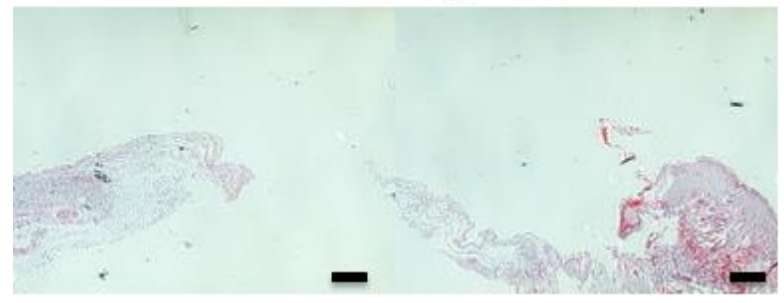

$0 \%$ CMP

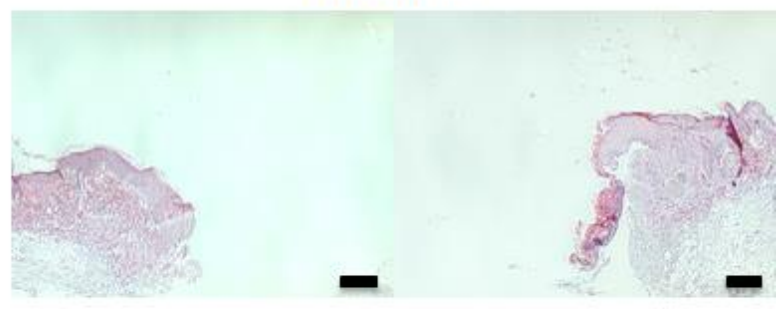

$20 \%$ CMP

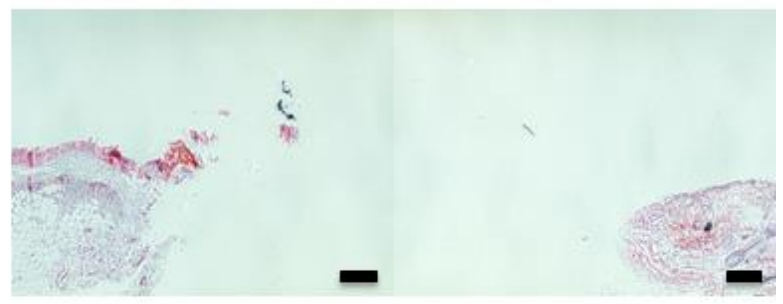

$50 \%$ CMP

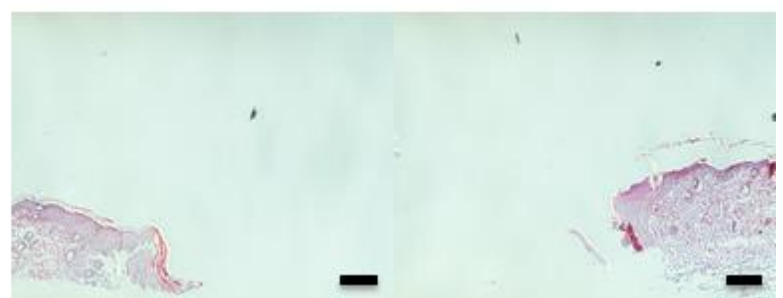

Figure S11: H\&E stained microscopy images of wound tissue sections at day 4 (scale bars: 200 $\mu \mathrm{m})$. The wound edges were far apart and could not be imaged at day 4 . Therefore, two images (one for each wound edge) were combined to show the wound area at day 4. The H\&E stained microscopy images of wound tissue sections at days 9 and 14 are presented in Figure 6 of the manuscript. 


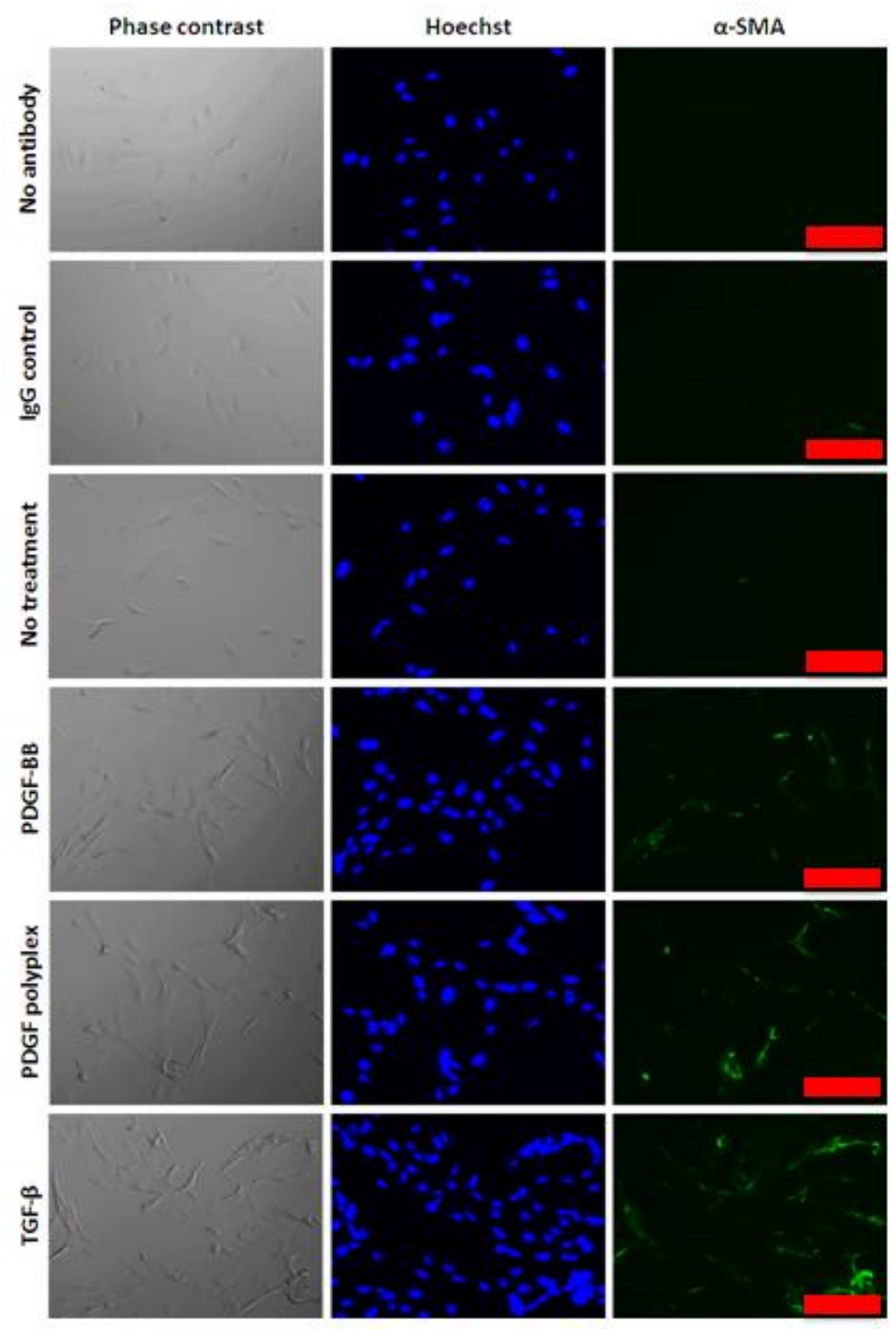

Figure S12: Determination of the effect of PDGF on myofibroblast activation within in vitro cultures (representative fluorescence microscopy images for $n=3$; scale bar: $100 \mu \mathrm{m}$ ). 


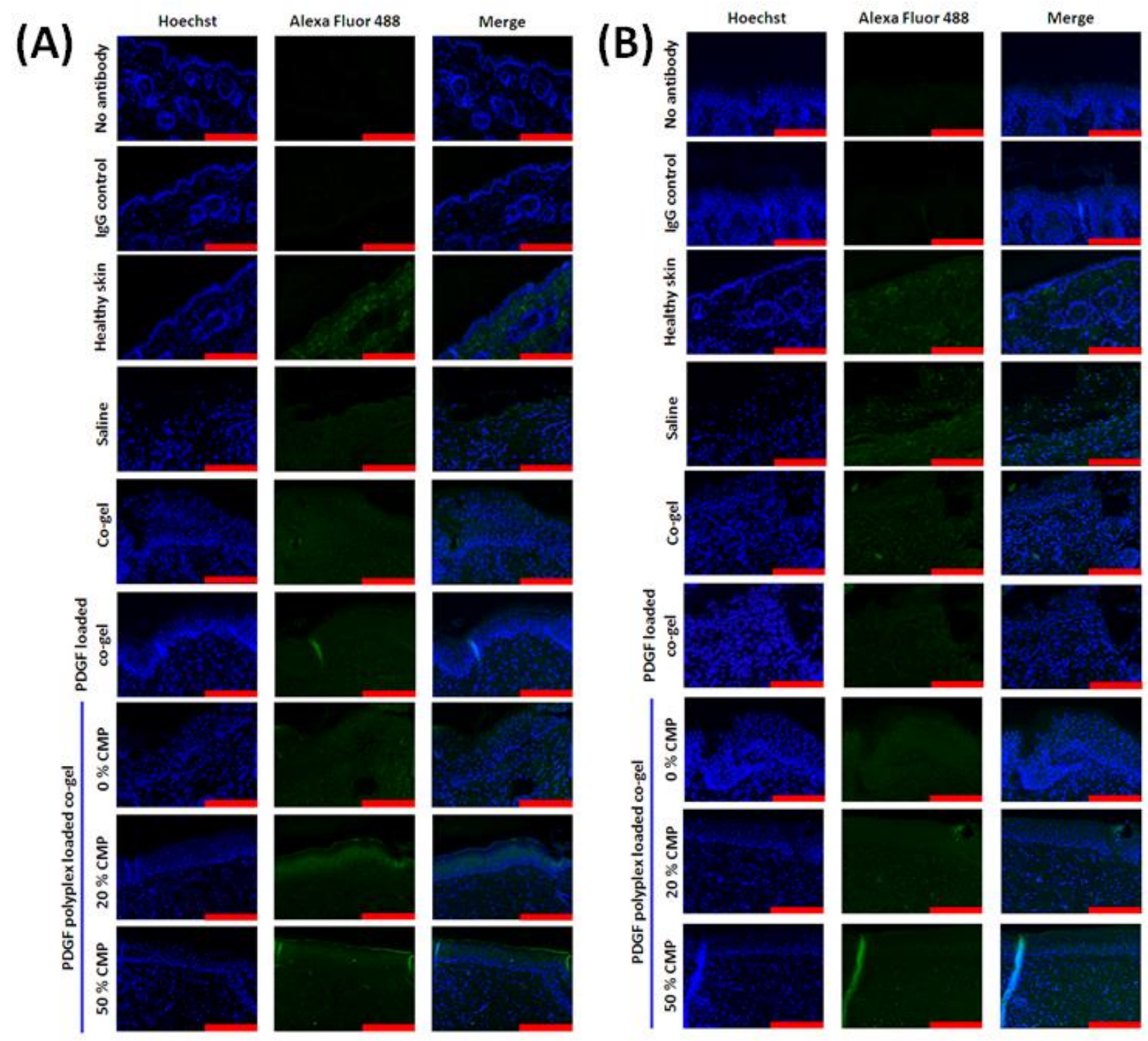

Figure S13: Visualization of collagen types in treated wounds. Representative fluorescence microscopy images for (A) collagen type I and (B) collagen type III for wound tissue sections treated with different formulations (scale bar: $250 \mu \mathrm{m}$ ). 